\title{
On the Global Dissipativity of a Class of Cellular Neural Networks with Multipantograph Delays
}

\author{
Liqun Zhou \\ Science of Mathematics College, Tianjin Normal University, Tianjin 300387, China \\ Correspondence should be addressed to Liqun Zhou, zhouliqun20000@163.com \\ Received 9 May 2011; Revised 23 September 2011; Accepted 8 October 2011 \\ Academic Editor: Tingwen Huang
}

Copyright () 2011 Liqun Zhou. This is an open access article distributed under the Creative Commons Attribution License, which permits unrestricted use, distribution, and reproduction in any medium, provided the original work is properly cited.

For the first time the global dissipativity of a class of cellular neural networks with multipantograph delays is studied. On the one hand, some delay-dependent sufficient conditions are obtained by directly constructing suitable Lyapunov functionals; on the other hand, firstly the transformation transforms the cellular neural networks with multipantograph delays into the cellular neural networks with constant delays and variable coefficients, and then constructing Lyapunov functionals, some delay-independent sufficient conditions are given. These new sufficient conditions can ensure global dissipativity together with their sets of attraction and can be applied to design global dissipative cellular neural networks with multipantograph delays and easily checked in practice by simple algebraic methods. An example is given to illustrate the correctness of the results.

\section{Introduction}

In recent years, cellular neural networks (CNNs) and delayed cellular neural networks (DCNNs) have been investigated widely because of their extensive applications in pattern recognition, image processing, association, synchronization problem, and many other fields. In such applications, it is of prime importance to ensure that the designed CNNs be stable. Therefore, the stability of CNNs with or without delay has received much attention (see, [1-10]). As pointed out in $[11,12]$, the global dissipativity is also an important concept in dynamical neural networks. The concept of global dissipativity in dynamical systems is a more general concept and it has found applications in the areas such as stability theory, chaos and synchronization theory, system norm estimation, and robust control $[11,12]$. Liao and Wang in [11] addressed the global dissipativity of a general class of continuous time recurrent neural networks and derived some sufficient conditions for the global dissipativity and global exponentially dissipativity. Arik in [12] analyzed the global dissipation of several classes of neural networks and derived some sufficient conditions for the global dissipativity of neural networks systems. To date, most research on
DCNNs has been restricted to simple cases of constant delays [1-4]. Some Papers that considered variable or distributed are those in $[5-7,9,10,13-15]$, and variable delays usually are considered to bounded delays. To the best of our knowledge, few authors have considered dynamical behavior for the CNNs with pantograph delays. Pantograph delay is also a kind of the objective existence, such as in Web quality of service (QoS). The pantograph delay systems as important mathematical models often arise in some fields such as physics, biology systems, and control theory. In this paper, our focus is on global dissipativity of multipantograph delayed cellular neural networks. The Lipschitz continuous activation functions are considered, and by constructing suitable Lyapunov functionals and applying matrix theory, some delay-dependent and delay-independent sufficient conditions are obtained. The main contributions of this paper include the derivations of new global attractive sets and characterization of global dissipativity. These properties play important roles in the design and applications of global dissipative CNNs with multipantograph delays, and are of great interest in many applications, such as chaos and synchronization theory, and robust control. 


\section{Model and Preliminaries}

Consider the model of multipantograph delayed CNNs described by the following functional differential equations:

$$
\begin{gathered}
\dot{x}_{i}(t)=-d_{i} x_{i}(t)+\sum_{j=1}^{n}\left[a_{i j} f_{j}\left(x_{j}(t)\right)+b_{i j} f_{j}\left(x_{j}\left(q_{1} t\right)\right)\right. \\
\left.+c_{i j} f_{j}\left(x_{j}\left(q_{2} t\right)\right)\right]+I_{i}, \\
x_{i}(0)=x_{i 0}, \quad i=1,2, \ldots, n,
\end{gathered}
$$

for $t>0$, where $x_{i}(t)$ denotes the potential (or voltage) of the cell $i$ at time $t ; f_{i}(\cdot)$ denotes a nonlinear activation function; $I_{i}$ denotes the $i$ th component of an external input source introduced from outside the network to the cell $i$ at time $t$; $d_{i}>0$ denotes the rate with which the cell $i$ resets its potential to the resting state when isolated from other cells and inputs at time $t ; a_{i j}, b_{i j}$, and $c_{i j}$ are constants which denote the strengths of connectivity between the cells $j$ and $i$ at time $t, q_{1} t$, and $q_{2} t$, respectively; $q_{1}, q_{2}$ are pantograph constants and satisfy $0<q_{1}, q_{2}<1$, and $q_{1} t=t-\left(1-q_{1}\right) t, q_{2} t=$ $t-\left(1-q_{2}\right) t$, in which $\left(1-q_{1}\right) t,\left(1-q_{2}\right) t$ correspond to the time delay required in processing and transmitting a signal from the $j$ th cell to the $i$ th cell; $x_{i 0}, i=1,2, \ldots, n$ is constant which denotes initial value of $x_{i}(t), i=1,2, \ldots, n$ at initial time $t=0$. In this paper, we consider the following Lipschitz continuous activation functions:

$$
\begin{gathered}
0 \leq \frac{f_{i}\left(x_{i}\right)-f_{i}\left(y_{i}\right)}{x_{i}-y_{i}} \leq l_{i}, \quad l_{i}>0, f_{i}(0)=0, \\
\left|f_{i}\left(x_{i}\right)\right| \longrightarrow \infty \quad \text { as }\left|x_{i}\right| \longrightarrow \infty,
\end{gathered}
$$

for all $x_{i}, y_{i} \in R, i=1,2, \ldots, n$, and denote $L=$ $\operatorname{diag}\left(l_{1}, l_{2}, \ldots, l_{n}\right)$.

The model (1) can be described in a vector form:

$$
\begin{gathered}
\dot{x}(t)=-D x(t)+A f(x(t))+B f\left(x\left(q_{1} t\right)\right)+C f\left(x\left(q_{2} t\right)\right)+I, \\
x(0)=x_{0},
\end{gathered}
$$

where $x=\left(x_{1}, x_{2}, \ldots, x_{n}\right)^{T}$ is the neuron state vector, $I=$ $\left(I_{1}, I_{2}, \ldots, I_{n}\right)^{T}$ is the bias vector, $D=\operatorname{diag}\left(d_{1}, d_{2}, \ldots, d_{n}\right)$, $A=\left(a_{i j}\right)_{n \times n}, B=\left(b_{i j}\right)_{n \times n}, C=\left(c_{i j}\right)_{n \times n}$ are connection weight matrices, $f(\cdot)=\left(f_{1}(\cdot), f_{2}(\cdot), \ldots, f_{n}(\cdot)\right)^{T}$ is a vectorvalued activation function, and $x_{0}=\left(x_{10}, x_{20}, \ldots, x_{n 0}\right)$ denotes initial value vector at initial time $t=0$.

Definition 1 (see [11]). The cellular neural network (1) is said to be a dissipative system, if there exists a compact set $S \subset R^{n}$, such that for all $x_{0} \in R^{n}$, there exist $t_{0}>0$, when $t \geq t_{0}, x\left(t, x_{0}\right) \subseteq S$, where $x\left(t, x_{0}\right)$ denotes the solution of (1) from initial state $x_{0}$ and initial time $t=0$. In this case, $S$ is called a globally attractive set. A set $S$ is called positive invariant if for all $x_{0} \in S$ implies $x\left(t, x_{0}\right) \subseteq S$ for $t \geq 0$. lows:

The induced matrix norms $\|\cdot\|_{p}$ are displayed as fol-

$$
\|A\|_{1}=\max _{j} \sum_{i=1}^{n}\left|a_{i j}\right|, \quad\|A\|_{\infty}=\max _{i} \sum_{j=1}^{n}\left|a_{i j}\right| .
$$

The transformation $y_{i}(t)=x_{i}\left(e^{t}\right)[16]$ transforms the neural networks (1) and (3), respectively, into the following cellular neural networks with constant time delays and variable coefficients:

$$
\begin{gathered}
\dot{y}_{i}(t)=e^{t}\left\{-d_{i} y_{i}(t)+\sum_{j=1}^{n}\left[a_{i j} f_{j}\left(y_{j}(t)\right)+b_{i j} f_{j}\left(y_{j}\left(t-\tau_{1}\right)\right)\right.\right. \\
\left.\left.+c_{i j} f_{j}\left(y_{j}\left(t-\tau_{2}\right)\right)\right]+I_{i}\right\} \\
y_{i}(s)=\varphi_{i}(s), \quad-\tau \leq s \leq 0, i=1,2 \ldots, n \\
\dot{y}(t)=e^{t}\left\{-D y(t)+A f(y(t))+B f\left(y\left(t-\tau_{1}\right)\right)\right. \\
\left.+C f\left(y\left(t-\tau_{2}\right)\right)+I\right\} \\
y(s)=\phi(s), \quad-\tau \leq s \leq 0
\end{gathered}
$$

where $\tau_{1}=-\log q_{1}>0, \tau_{2}=-\log q_{2}>0, \tau=\max \left\{\tau_{1}, \tau_{2}\right\}$, $\phi(s)=\left(\varphi_{1}(s), \varphi_{2}(s), \ldots, \varphi_{n}(s)\right)^{T}$, in which $\varphi_{i} \in C([-\tau, 0], R)$, $i=1,2, \ldots, n$ are continuous functions.

Remark 2. The models (1), (3), (5), and (6) of this paper are different from the models in $[11,12]$. The models in $[11,12]$ are neural networks with constant delays, but in this paper the model (1) or (3) is the cellular neural network with multipantograph delays which are unbounded functions; the model (5) or (6) is the cellular neural network with constant delays and variable coefficients. The results in $[11,12]$ cannot be applied to the models (1), (3), (5), and (6) in this paper. Therefore, our results establish new criteria for the global dissipativity of cellular neural networks with multipantograph delays.

Definition 3 (see [17]). The cellular neural network (5) is said to be a dissipative system, if there exists a compact set $S \subset R^{n}$ such that for any compact set $\phi \subset R^{n}$, there exist $t_{0}=t_{0}(\phi)$, when $t \geq t_{0}, y(t, \phi) \subseteq S$, where $y(t, \phi)$ denotes the solution of (5) from initial state $\phi$. In this case, $S$ is called a globally attractive set. A set $S$ is called positive invariant if for all $\phi \in S$ implies $y(t, \phi) \subseteq S$ for $t \geq t_{0}$.

Lemma 4. For any vectors $a, b \in R^{n}$, the inequality

$$
2 a^{T} b \leq a^{T} X a+b^{T} X b
$$

holds, in which $X$ is any matrix with $X>0$.

\section{Main Results}

Theorem 5. If condition (2) is satisfied and the following condition holds:

$$
A+A^{T}+B B^{T}+C C^{T}+\left(\frac{1}{q_{1}}+\frac{1}{q_{2}}\right) I \leq 0,
$$

then the cellular neural network (3) is a dissipative system and the set $S_{1}=\left\{x|| f_{i}\left(x_{i}(t)\right)\left|\leq l_{i}\right| I_{i} \mid / d_{i}, i=1,2, \ldots, n\right\}$ is a positive invariant and globally attractive set. 
Proof. The following positive definite and radially unbounded Lyapunov functional will be used:

$$
\begin{aligned}
V(x(t))= & 2 \sum_{i=1}^{n} \int_{0}^{x_{i}(t)} f_{i}(s) d s+\sum_{i=1}^{n} \int_{q_{1} t}^{t} \frac{1}{q_{1}} f_{i}^{2}\left(x_{i}(\zeta)\right) d \zeta \\
& +\sum_{i=1}^{n} \int_{q_{2} t}^{t} \frac{1}{q_{2}} f_{i}^{2}\left(x_{i}(\eta)\right) d \eta .
\end{aligned}
$$

The time derivative of $V(x(t))$ along the trajectories of the system (1) is obtained as follows:

$$
\begin{aligned}
\dot{V}(x(t))= & -2 \sum_{i=1}^{n} d_{i} f_{i}\left(x_{i}(t)\right) x_{i}(t)+2 \sum_{i=1}^{n} \sum_{j=1}^{n} a_{i j} f_{i}\left(x_{i}(t)\right) f_{j}\left(x_{j}(t)\right) \\
& +2 \sum_{i=1}^{n} \sum_{j=1}^{n} b_{i j} f_{i}\left(x_{i}(t)\right) f_{j}\left(x_{j}\left(q_{1} t\right)\right) \\
& +2 \sum_{i=1}^{n} \sum_{j=1}^{n} c_{i j} f_{i}\left(x_{i}(t)\right) f_{j}\left(x_{j}\left(q_{2} t\right)\right)+2 \sum_{i=1}^{n} f_{i}\left(x_{i}(t)\right) I_{i} \\
& +\sum_{i=1}^{n} \frac{1}{q_{1}} f_{i}^{2}\left(x_{i}(t)\right)-\sum_{i=1}^{n} f_{i}^{2}\left(x_{i}\left(q_{1} t\right)\right) \\
& +\sum_{i=1}^{n} \frac{1}{q_{2}} f_{i}^{2}\left(x_{i}(t)\right)-\sum_{i=1}^{n} f_{i}^{2}\left(x_{i}\left(q_{2} t\right)\right) \\
\leq & -2 \sum_{i=1}^{n} \frac{d_{i}}{l_{i}} f_{i}^{2}\left(x_{i}(t)\right)+2 \sum_{i=1}^{n}\left|f_{i}\left(x_{i}(t)\right)\right|\left|I_{i}\right| \\
& +f^{T}(x(t))\left(A+A^{T}\right) f(x(t)) \\
& +2 f^{T}\left(x\left(q_{1} t\right)\right) B^{T} f(x(t)) \\
& +2 f^{T}\left(x\left(q_{2} t\right)\right) C^{T} f(x(t)) \\
& \left(x\left(q_{1} t\right)\right) f\left(x\left(q_{1} t\right)\right)-f^{T}\left(x\left(q_{2} t\right)\right) f\left(x\left(q_{2} t\right)\right) . \\
q_{2} & f^{T}(x(t)) f(x(t)) \\
& \\
& +10) \\
& \\
&
\end{aligned}
$$

In view of Lemma 4, we obtain

$$
\begin{aligned}
& -f^{T}\left(x\left(q_{1} t\right)\right) f\left(x\left(q_{1} t\right)\right)+2 f^{T}\left(x\left(q_{1} t\right)\right) B^{T} f(x(t)) \\
& \quad \leq f^{T}(x(t)) B B^{T} f(x(t)), \\
& -f^{T}\left(x\left(q_{2} t\right)\right) f\left(x\left(q_{2} t\right)\right)+2 f^{T}\left(x\left(q_{2} t\right)\right) C^{T} f(x(t)) \\
& \quad \leq f^{T}(x(t)) C C^{T} f(x(t)) .
\end{aligned}
$$

Using the above inequality in (10) results in

$$
\begin{aligned}
\dot{V}(x(t)) \leq & -2 \sum_{i=1}^{n} \frac{d_{i}}{l_{i}} f_{i}^{2}\left(x_{i}(t)\right)+2 \sum_{i=1}^{n}\left|f_{i}\left(x_{i}(t)\right)\right|\left|I_{i}\right| \\
& +f^{T}(x(t))\left\{A+A^{T}+B B^{T}+C C^{T}\right. \\
& \left.+\left(\frac{1}{q_{1}}+\frac{1}{q_{2}}\right) I\right\} f(x(t))<0,
\end{aligned}
$$

for $x_{i} \in R^{n} \backslash S_{1}$, implying that the set $S_{1}$ is a positive invariant and globally attractive set.

Theorem 6. If condition (2) is satisfied and the following condition holds:

$$
A+A^{T}+B B^{T}+C C^{T}+2 I \leq 0,
$$

then the cellular neural network (6) is a dissipative system and the set $S_{2}=\left\{y|| f_{i}\left(y_{i}(t)\right)\left|\leq l_{i}\right| I_{i} \mid / d_{i}, i=1,2, \ldots, n\right\}$ is a positive invariant and globally attractive set.

Proof. The following positive definite and radially unbounded Lyapunov functional will be used:

$$
\begin{aligned}
V(t, y(t))= & 2 \sum_{i=1}^{n} e^{-t} \int_{0}^{y_{i}(t)} f_{i}(s) d s+\sum_{i=1}^{n} \int_{t-\tau_{1}}^{t} f_{i}^{2}\left(y_{i}(\zeta)\right) d \zeta \\
& +\sum_{i=1}^{n} \int_{t-\tau_{2}}^{t} f_{i}^{2}\left(y_{i}(\eta)\right) d \eta .
\end{aligned}
$$

The time derivative of $V(y(t))$ along the trajectories of the system (5) is obtained as follows:

$$
\begin{aligned}
\dot{V}(t, y(t))= & -2 \sum_{i=1}^{n} e^{-t} \int_{0}^{y_{i}(t)} f_{i}(s) d s-2 \sum_{i=1}^{n} d_{i} f_{i}\left(y_{i}(t)\right) y_{i}(t) \\
& +2 \sum_{i=1}^{n} \sum_{j=1}^{n} a_{i j} f_{i}\left(y_{i}(t)\right) f_{j}\left(y_{j}(t)\right) \\
& +2 \sum_{i=1}^{n} \sum_{j=1}^{n} b_{i j} f_{i}\left(y_{i}(t)\right) f_{j}\left(y_{j}\left(t-\tau_{1}\right)\right) \\
& +2 \sum_{i=1}^{n} \sum_{j=1}^{n} c_{i j} f_{i}\left(y_{i}(t)\right) f_{j}\left(y_{j}\left(t-\tau_{2}\right)\right) \\
& +2 \sum_{i=1}^{n} f_{i}\left(y_{i}(t)\right) I_{i}+\sum_{i=1}^{n} f_{i}^{2}\left(y_{i}(t)\right) \\
& -\sum_{i=1}^{n} f_{i}^{2}\left(y_{i}\left(t-\tau_{1}\right)\right)+\sum_{i=1}^{n} f_{i}^{2}\left(y_{i}(t)\right) \\
& -\sum_{i=1}^{n} f_{i}^{2}\left(y_{i}\left(t-\tau_{2}\right)\right)
\end{aligned}
$$




$$
\begin{aligned}
\leq & -2 \sum_{i=1}^{n} \frac{d_{i}}{l_{i}} f_{i}^{2}\left(y_{i}(t)\right)+2 \sum_{i=1}^{n}\left|f_{i}\left(y_{i}(t)\right)\right|\left|I_{i}\right| \\
& +f^{T}(y(t))\left(A+A^{T}\right) f(y(t)) \\
& +2 f^{T}\left(y\left(t-\tau_{1}\right)\right) B^{T} f(y(t)) \\
& +2 f^{T}\left(y\left(t-\tau_{2}\right)\right) C^{T} f(y(t)) \\
& +2 f^{T}(y(t)) f(y(t)) \\
& -f^{T}\left(y\left(t-\tau_{1}\right)\right) f\left(y\left(t-\tau_{1}\right)\right) \\
& -f^{T}\left(y\left(t-\tau_{2}\right)\right) f\left(y\left(t-\tau_{2}\right)\right) \\
\leq & -2 \sum_{i=1}^{n} \frac{d_{i}}{l_{i}} f_{i}^{2}\left(y_{i}(t)\right)+2 \sum_{i=1}^{n}\left|f_{i}\left(y_{i}(t)\right)\right|\left|I_{i}\right| \\
& +f^{T}(y(t)) \\
& \times\left(A+A^{T}+B B^{T}+C C^{T}+2 I\right) f(y(t)) \\
< & 0, \quad \text { for } y_{i} \in R^{n} \backslash S_{2} .
\end{aligned}
$$

So the set $S_{2}$ is a positive invariant and globally attractive set.

Corollary 7. If condition (2) is satisfied and the following condition holds:

$$
A+A^{T}+B B^{T}+C C^{T}+2 I \leq 0
$$

then the cellular neural network (3) is a dissipative system and the set $S_{3}=\left\{x|| f_{i}\left(x_{i}\left(e^{t}\right)\right)\left|\leq l_{i}\right| I_{i} \mid / d_{i}, i=1,2, \ldots, n\right\}$ is a positive invariant and globally attractive set.

Theorem 8. If condition (2) is satisfied and the matrix $Q$ given by

$$
\begin{aligned}
Q= & P\left(A-L^{-1} D\right)+\left(A-L^{-1} D\right)^{T} P+P B B^{T} P \\
& +P C C^{T} P+\left(\frac{1}{q_{1}}+\frac{1}{q_{2}}\right) I
\end{aligned}
$$

is negative definite, then the cellular neural network (3) is a dissipative system and the set

$$
\begin{gathered}
S_{4}=\left\{x \mid \sum_{i=1}^{n}\left(f_{i}\left(x_{i}(t)\right)+\frac{p_{i} I_{i}}{\lambda_{M}(Q)}\right)^{2} \leq \sum_{i=1}^{n}\left(\frac{p_{i} I_{i}}{\lambda_{M}(Q)}\right)^{2},\right. \\
i=1,2, \ldots, n\}
\end{gathered}
$$

is a positive invariant and globally attractive set, where $L=$ $\operatorname{diag}\left(l_{1}, l_{2}, \ldots, l_{n}\right), P=\operatorname{diag}\left(p_{1}, p_{2}, \ldots, p_{n}\right)$, and $\lambda_{M}(Q)$ is the maximum eigenvalue of the matrix $Q$.
Proof. Let us employ the following positive definite and radially unbounded Lyapunov functional:

$$
\begin{aligned}
V(x(t))= & 2 \sum_{i=1}^{n} p_{i} \int_{0}^{x_{i}(t)} f_{i}(s) d s+\sum_{i=1}^{n} \int_{q_{1} t}^{t} \frac{1}{q_{1}} f_{i}^{2}\left(x_{i}(\zeta)\right) d \zeta \\
& +\sum_{i=1}^{n} \int_{q_{2} t}^{t} \frac{1}{q_{2}} f_{i}^{2}\left(x_{i}(\eta)\right) d \eta .
\end{aligned}
$$

The time derivative of $V(x(t))$ along the trajectories of the system (1) is obtained as follows:

$$
\begin{aligned}
\dot{V}(x(t))= & -2 \sum_{i=1}^{n} p_{i} d_{i} f_{i}\left(x_{i}(t)\right) x_{i}(t) \\
& +2 \sum_{i=1}^{n} \sum_{j=1}^{n} p_{i} a_{i j} f_{i}\left(x_{i}(t)\right) f_{j}\left(x_{j}(t)\right) \\
& +2 \sum_{i=1}^{n} \sum_{j=1}^{n} p_{i} b_{i j} f_{i}\left(x_{i}(t)\right) f_{j}\left(x_{j}\left(q_{1} t\right)\right) \\
& +2 \sum_{i=1}^{n} \sum_{j=1}^{n} p_{i} c_{i j} f_{i}\left(x_{i}(t)\right) f_{j}\left(x_{j}\left(q_{2} t\right)\right) \\
& +2 \sum_{i=1}^{n} p_{i} f_{i}\left(x_{i}(t)\right) I_{i}+\sum_{i=1}^{n} \frac{1}{q_{1}} f_{i}^{2}\left(x_{i}(t)\right) \\
& -\sum_{i=1}^{n} f_{i}^{2}\left(x_{i}\left(q_{1} t\right)\right) \\
& +\sum_{i=1}^{n} \frac{1}{q_{1}} f_{i}^{2}\left(x_{i}(t)\right)-\sum_{i=1}^{n} f_{i}^{2}\left(x_{i}\left(q_{2} t\right)\right) \\
\leq & -2 \sum_{i=1}^{n} p_{i} \frac{d_{i}}{l_{i}} f_{i}^{2}\left(x_{i}(t)\right)+2 \sum_{i=1}^{n} p_{i} f_{i}\left(x_{i}(t)\right) I_{i} \\
& +f^{T}(x(t))\left(P A+A^{T} P\right) f(x(t)) \\
& +2 f^{T}\left(x\left(q_{1} t\right)\right) B^{T} P f(x(t)) \\
& \left.+2 f^{T}\left(x\left(x_{i}(t)\right) I_{i} t\right)\right) C^{T} P f(x(t)) \\
& +\left(\frac{1}{q_{1}}+\frac{1}{q_{1}}\right) f^{T}(x(t)) f(x(t)) \\
& f^{T}\left(x\left(q_{1} t\right)\right) f\left(x\left(q_{1} t\right)\right)-f^{T}\left(x\left(q_{2} t\right)\right) f\left(x\left(q_{2} t\right)\right) \\
& \left.(A)+\left(A-L^{-1} D\right)^{T} P\right) f(x(t)) \\
&
\end{aligned}
$$




$$
\begin{aligned}
& +2 f^{T}\left(x\left(q_{1} t\right)\right) B^{T} P f(x(t)) \\
& +2 f^{T}\left(x\left(q_{2} t\right)\right) C^{T} P f(x(t)) \\
& +\left(\frac{1}{q_{1}}+\frac{1}{q_{1}}\right) f^{T}(x(t)) f(x(t)) \\
& -f^{T}\left(x\left(q_{1} t\right)\right) f\left(x\left(q_{1} t\right)\right)-f^{T}\left(x\left(q_{2} t\right)\right) f\left(x\left(q_{2} t\right)\right) .
\end{aligned}
$$

By Lemma 4, we obtain

$$
\begin{aligned}
& -f^{T}\left(x\left(q_{1} t\right)\right) f\left(x\left(q_{1} t\right)\right)+2 f^{T}\left(x\left(q_{1} t\right)\right) B^{T} P f(x(t)) \\
& \quad \leq f^{T}(x(t)) P B B^{T} P f(x(t)), \\
& -f^{T}\left(x\left(q_{2} t\right)\right) f\left(x\left(q_{2} t\right)\right)+2 f^{T}\left(x\left(q_{2} t\right)\right) C^{T} P f(x(t)) \\
& \quad \leq f^{T}(x(t)) P C C^{T} P f(x(t)) .
\end{aligned}
$$

Using the above inequality in (20) results in

$$
\begin{aligned}
\dot{V}(x(t)) \leq & 2 \sum_{i=1}^{n} p_{i} f_{i}\left(x_{i}(t)\right) I_{i}+f^{T}(x(t)) \\
& \times\left(P\left(A-L^{-1} D\right)+\left(A-L^{-1} D\right)^{T} P\right) f(x(t)) \\
& +\left(\frac{1}{q_{1}}+\frac{1}{q_{1}}\right) f^{T}(x(t)) f(x(t)) \\
& +f^{T}(x(t)) P B B^{T} P f(x(t)) \\
& +f^{T}(x(t)) P C C^{T} P f(x(t)) \\
= & 2 \sum_{i=1}^{n} p_{i} f_{i}\left(x_{i}(t)\right) I_{i}+f^{T}(x(t)) Q f(x(t)) \\
\leq & 2 \sum_{i=1}^{n} p_{i} f_{i}\left(x_{i}(t)\right) I_{i}+\sum_{i=1}^{n} \lambda_{M}(Q) f_{i}^{2}\left(x_{i}(t)\right) \\
= & \lambda_{M}(Q) \sum_{i=1}^{n}\left\{\left(f_{i}\left(x_{i}(t)\right)+\frac{p_{i} I_{i}}{\lambda_{M}(Q)}\right)^{2}-\left(\frac{p_{i} I_{i}}{\lambda_{M}(Q)}\right)^{2}\right\} \\
< & 0, \quad \text { when } f_{i}\left(x_{i}\right) \in R^{n} \backslash S_{4},
\end{aligned}
$$

implying that the set $S_{4}$ is a positive invariant and globally attractive set.

Theorem 9. If condition (2) is satisfied and the matrix $Q$ given by

$$
\begin{aligned}
Q= & P\left(A-L^{-1} D\right)+\left(A-L^{-1} D\right)^{T} P+P B B^{T} P \\
& +P C C^{T} P+2 I
\end{aligned}
$$

is negative definite, then the cellular neural network (6) is a dissipative system and the set

$$
\begin{gathered}
S_{5}=\left\{y \mid \sum_{i=1}^{n}\left(f_{i}\left(y_{i}(t)\right)+\frac{p_{i} I_{i}}{\lambda_{M}(Q)}\right)^{2} \leq \sum_{i=1}^{n}\left(\frac{p_{i} I_{i}}{\lambda_{M}(Q)}\right)^{2},\right. \\
i=1,2, \ldots, n\}
\end{gathered}
$$

is a positive invariant and globally attractive set where $P=$ $\operatorname{diag}\left(p_{1}, p_{2}, \ldots, p_{n}\right)$, and $\lambda_{M}(Q)$ is the maximum eigenvalue of the matrix $Q$.

Proof. Let us employ the following positive definite and radially unbounded Lyapunov functional:

$$
\begin{aligned}
V(t, y(t))= & -2 \sum_{i=1}^{n} p_{i} e^{-t} \int_{0}^{y_{i}(t)} f_{i}(s) d s+\sum_{i=1}^{n} \int_{t-\tau_{1}}^{t} f_{i}^{2}\left(y_{i}(\zeta)\right) d \zeta \\
& +\sum_{i=1}^{n} \int_{t-\tau_{2}}^{t} f_{i}^{2}\left(y_{i}(\eta)\right) d \eta .
\end{aligned}
$$

The time derivative of $V(y(t))$ along the trajectories of the system (5) is obtained as follows:

$$
\begin{aligned}
\dot{V}(t, y(t))= & -2 \sum_{i=1}^{n} p_{i} e^{-t} \int_{0}^{y_{i}(t)} f_{i}(s) d s \\
& -2 \sum_{i=1}^{n} p_{i} d_{i} f_{i}\left(y_{i}(t)\right) y_{i}(t) \\
& +2 \sum_{i=1}^{n} \sum_{j=1}^{n} p_{i} a_{i j} f_{i}\left(y_{i}(t)\right) f_{j}\left(y_{j}(t)\right) \\
& +2 \sum_{i=1}^{n} \sum_{j=1}^{n} p_{i} b_{i j} f_{i}\left(y_{i}(t)\right) f_{j}\left(y_{j}\left(t-\tau_{1}\right)\right) \\
& +2 \sum_{i=1}^{n} \sum_{j=1}^{n} p_{i} c_{i j} f_{i}\left(y_{i}(t)\right) f_{j}\left(y_{j}\left(t-\tau_{2}\right)\right) \\
& +2 \sum_{i=1}^{n} p_{i} f_{i}\left(y_{i}(t)\right) I_{i}+\sum_{i=1}^{n} f_{i}^{2}\left(y_{i}(t)\right) \\
& -\sum_{i=1}^{n} f_{i}^{2}\left(y_{i}\left(t-\tau_{1}\right)\right)+\sum_{i=1}^{n} f_{i}^{2}\left(y_{i}(t)\right) \\
& -\sum_{i=1}^{n} f_{i}^{2}\left(y_{i}\left(t-\tau_{2}\right)\right) \\
\leq & -2 \sum_{i=1}^{n} \frac{d_{i}}{l_{i}} f_{i}^{2}\left(y_{i}(t)\right)+2 \sum_{i=1}^{n} p_{i} f_{i}\left(y_{i}(t)\right) I_{i} \\
& +f^{T}(y(t))\left(P A+A^{T} P\right) f(y(t))
\end{aligned}
$$




$$
\begin{aligned}
& +2 f^{T}\left(y\left(t-\tau_{1}\right)\right) B^{T} P f(y(t)) \\
& +2 f^{T}\left(y\left(t-\tau_{2}\right)\right) C^{T} P f(y(t)) \\
& +2 f^{T}(y(t)) f(y(t)) \\
& -f^{T}\left(y\left(t-\tau_{1}\right)\right) f\left(x\left(t-\tau_{1}\right)\right) \\
& -f^{T}\left(x\left(t-\tau_{2}\right)\right) f\left(x\left(t-\tau_{2}\right)\right) \\
= & 2 \sum_{i=1}^{n} p_{i} f_{i}\left(y_{i}(t)\right) I_{i}+f^{T}(y(t)) \\
& \times\left(P\left(A-L^{-1} D\right)+\left(A-L^{-1} D\right)^{T} P\right) f(y(t)) \\
& +2 f^{T}\left(y\left(t-\tau_{1}\right)\right) B^{T} P f(y(t)) \\
& +2 f^{T}\left(y\left(t-\tau_{2}\right)\right) C^{T} P f(y(t)) \\
& +2 f^{T}(y(t)) f(y(t)) \\
& -f^{T}\left(y\left(t-\tau_{1}\right)\right) f\left(y\left(t-\tau_{1}\right)\right) \\
& -f^{T}\left(y\left(t-\tau_{2}\right)\right) f\left(y\left(t-\tau_{2}\right)\right) \\
\leq & 2 \sum_{i=1}^{n} p_{i} f_{i}\left(y_{i}(t)\right) I_{i}+f^{T}(y(t)) \\
& \times\left(P\left(A-L^{-1} D\right)+\left(A-L^{-1} D\right)^{T} P\right) f(y(t)) \\
& +\sum_{i=1}^{n}\left\{\left(f_{i}\left(y_{i}(t)\right)+\frac{p_{i} I_{i}}{\lambda_{M}(Q)}\right)^{2}-\left(\frac{p_{i} I_{i}}{\lambda_{M}(Q)}\right)^{2}\right\} \\
\leq & \left.2 \sum_{i=1}^{n} p_{i} f_{i}\left(y_{i}\right) \in R^{n} \backslash S_{5}(t)\right) I_{i}+f^{T}(y(t)) Q f(x(t)) \\
& +2 f_{i}^{T}(y(t)) f(y(t)) \\
& +f^{T}(y(t)) P B B^{T} P f(y(t)) \\
& +f^{T}(y(t)) P C C^{T} P f(y(t)) \\
& \lambda_{i}(Q) \lambda_{M}(Q) f_{i}^{2}\left(y_{i}(t)\right) \\
& (y=1 \\
&
\end{aligned}
$$

So the set $S_{5}$ is a positive invariant and globally attractive set.

Corollary 10. If condition (2) is satisfied and the matrix $Q$ given by

$$
\begin{aligned}
Q= & P\left(A-L^{-1} D\right)+\left(A-L^{-1} D\right)^{T} P+P B B^{T} P \\
& +P C C^{T} P+2 I
\end{aligned}
$$

is negative definite, then the cellular neural network (3) is a dissipative system and the set

$$
\begin{gathered}
S_{6}=\left\{x \mid \sum_{i=1}^{n}\left(f_{i}\left(x_{i}\left(e^{t}\right)\right)+\frac{p_{i} I_{i}}{\lambda_{M}(Q)}\right)^{2} \leq \sum_{i=1}^{n}\left(\frac{p_{i} I_{i}}{\lambda_{M}(Q)}\right)^{2},\right. \\
i=1,2, \ldots, n\}
\end{gathered}
$$

is a positive invariant and globally attractive set, where $L=$ $\operatorname{diag}\left(l_{1}, l_{2}, \ldots, l_{n}\right), P=\operatorname{diag}\left(p_{1}, p_{2}, \ldots, p_{n}\right)$, and $\lambda_{M}(Q)$ is the maximum eigenvalue of the matrix $Q$.

Remark 11. The above theorems and corollaries imply that the equilibrium of a neural network only lies in the positive invariant and globally attractive set, any properties of activation function that satisfied (2) can be utilized, and the condition using the Lasalle invariant principle is given.

\section{Examples}

Example 12. Consider the following system:

$$
\begin{aligned}
\left(\begin{array}{l}
\dot{x}_{1}(t) \\
\dot{x}_{2}(t)
\end{array}\right)= & -\left(\begin{array}{ll}
\frac{1}{3} & 0 \\
0 & \frac{1}{4}
\end{array}\right)\left(\begin{array}{l}
x_{1}(t) \\
x_{2}(t)
\end{array}\right)+\left(\begin{array}{cc}
-5 & -10 \\
10 & -5
\end{array}\right)\left(\begin{array}{l}
f_{1}\left(x_{1}(t)\right) \\
f_{2}\left(x_{2}(t)\right)
\end{array}\right) \\
& +\left(\begin{array}{ll}
1 & 1 \\
1 & 1
\end{array}\right)\left(\begin{array}{l}
f_{1}\left(x_{1}\left(q_{1} t\right)\right) \\
f_{2}\left(x_{2}\left(q_{1} t\right)\right)
\end{array}\right) \\
& +\left(\begin{array}{cc}
-1 & 1 \\
1 & -1
\end{array}\right)\left(\begin{array}{l}
f_{1}\left(x_{1}\left(q_{2} t\right)\right) \\
f_{2}\left(x_{2}\left(q_{2} t\right)\right)
\end{array}\right)+\left(\begin{array}{l}
2 \\
3
\end{array}\right) .
\end{aligned}
$$

The activation functions $f_{1}\left(x_{1}\right)=\sin \left((1 / 3) x_{1}\right)+(1 / 3) x_{1}$, $f_{2}\left(x_{2}\right)=\cos \left((1 / 2) x_{2}\right)+(1 / 4) x_{2}$ are obviously Lipschitz continuous with Lipschitz constant $l_{1}=2 / 3, l_{2}=3 / 4$, and the pantograph coefficient is $q_{1}=1 / 2, q_{1}=3 / 4$. By some simple calculations, we obtain

$$
A+A^{T}+B B^{T}+C C^{T}+\left(2+\frac{4}{3}\right) I=\left(\begin{array}{cc}
-\frac{8}{3} & 0 \\
0 & -\frac{8}{3}
\end{array}\right)
$$

is negative definite. According to Theorem 5, the pantograph delay cellular neural network (29) is a dissipative system with a globally attractive set

$$
S=\left\{x|| f_{1}\left(x_{1}\right)\left|\leq \frac{l_{1}\left|I_{1}\right|}{d_{1}}=4,\right| f_{2}\left(x_{2}\right) \mid \leq \frac{l_{2}\left|I_{2}\right|}{d_{2}}=9\right\} .
$$

\section{Conclusions}

The pantograph delay systems as an important mathematical models often arise in some fields such as physics, biology systems, and control theory. In this paper, we firstly have studied 
the global dissipativity of a class of cellular neural networks with multipantograph delays. By using Lyapunov functional and matrix theory, we obtain some delay-dependent and delay-independent sufficient conditions which characterize global dissipation with their sets of attraction, which might have an impact in studying the uniqueness of equilibria, globally asymptotic stability, instability, and the existence of periodic solutions. And these sufficient conditions are easily checked in practice by simple algebraic methods. Remarkbly, our results hold for the classes of neural networks that are different from those considered in $[11,12]$. One example is given to illustrate the correctness of our results.

\section{Acknowledgments}

This work is supported by the NSF of China (no. 60974144), Science and Technology Development Foundation of Tianjin Colleges and Universities (no. 20100813) and Foundation for Doctors of Tianjin Normal University (no. 52LX34).

\section{References}

[1] L. O. Chua and L. Yang, "Cellular neural networks: theory and applications," IEEE Transactions on Circuits and Systems I, vol. 35, no. 10, pp. 1257-1290, 1998.

[2] T. Roska and L. O. Chua, "Cellular neural networks with nonlinear and delay-type template elements and non-uniform grids," International Journal of Circuit Theory and Applications, vol. 20, no. 4, pp. 469-481, 1992.

[3] N. Takahashi, "A new sufficient condition for complete stability of cellular neural networks with delay," IEEE Transactions on Circuits and Systems I, vol. 47, no. 6, pp. 793-799, 2000.

[4] S. Mohamad and K. Gopalsamy, "Exponential stability of continuous-time and discrete-time cellular neural networks with delays," Applied Mathematics and Computation, vol. 135, no. 1, pp. 17-38, 2003.

[5] Y. Zhang, A. H. Pheng, and S. L. Kwong, "Convergence analysis of cellular neural networks with unbounded delay," IEEE Transactions on Circuits and Systems I, vol. 48, no. 6, pp. 680$687,2001$.

[6] C. Sun and L. Li, "Dynamics of general neural networks with distributed delays," in Proceeding of the 3rd International Symposium on Neural Networks, vol. 3971, part I of Lecture Notes in Computer Science, pp. 135-140, Chengdu, China, May 2006.

[7] X. X. Liao and J. Wang, "Algebraic criteria for global exponential stability of cellular neural networks with multiple time delays," IEEE Transactions on Circuits and Systems I, vol. 50, no. 2, pp. 268-275, 2003.

[8] S. Arik and V. Tavsanoglu, "On the global asymptotic stability of delayed cellular neural networks," IEEE Transactions on Circuits and Systems I, vol. 47, no. 4, pp. 571-574, 2000.

[9] Y. Yang and J. Cao, "Stability and periodicity in delayed cellular neural networks with impulsive effects," Nonlinear Analysis, vol. 8, no. 1, pp. 362-374, 2007.

[10] C. Huang, L. Huang, and Z. Yuan, "Global stability analysis of a class of delayed cellular neural networks," Mathematics and Computers in Simulation, vol. 70, no. 3, pp. 133-148, 2005.

[11] X. Liao and J. Wang, "Global dissipativity of continuous-time recurrent neural networks with time delay," Physical Review E, vol. 68, no. 1, Article ID 016118, pp. 1-7, 2003.
[12] S. Arik, "On the global dissipativity of dynamical neural networks with time delays," Physics Letters A, vol. 326, no. 1-2, pp. 126-132, 2004.

[13] Z. Zeng and J. Wang, "Multiperiodicity and exponential attractivity evoked by periodic external inputs in delayed cellular neural networks," Neural Computation, vol. 18, no. 4, pp. 848870, 2006.

[14] W. Su and Y. Chen, "Global asymptotic stability analysis for neutral stochastic neural networks with time-varying delays," Communications in Nonlinear Science and Numerical Simulation, vol. 14, no. 4, pp. 1576-1581, 2009.

[15] L. Zhou and G. Hu, "Global exponential periodicity and stability of cellular neural networks with variable and distributed delays," Applied Mathematics and Computation, vol. 195, no. 2, pp. 402-411, 2008.

[16] Y. K. Liu, "Asymptotic behavior of functional differential equations with priportional time delays," European Journal of Applied Mathematics, vol. 7, pp. 11-30, 1996.

[17] C. Huang, "Dissipativity of Runge-Kutta methods for dynamical systems with delays," IMA Journal of Numerical Analysis, vol. 20, no. 1, pp. 153-166, 2000. 

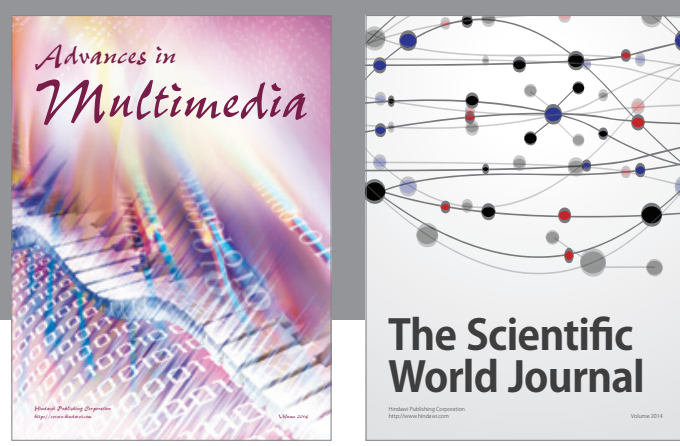

The Scientific World Journal
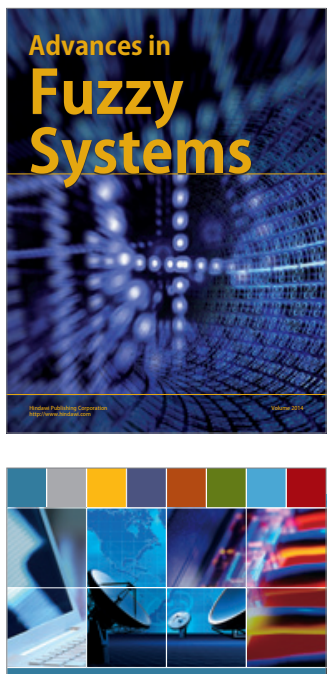

Computer Networks and Communications
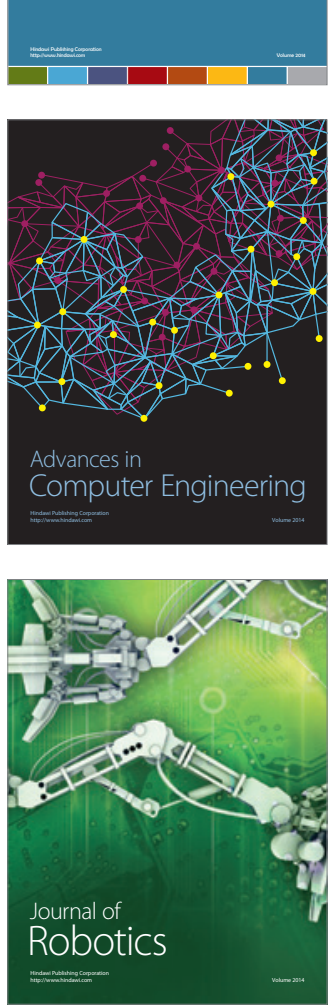
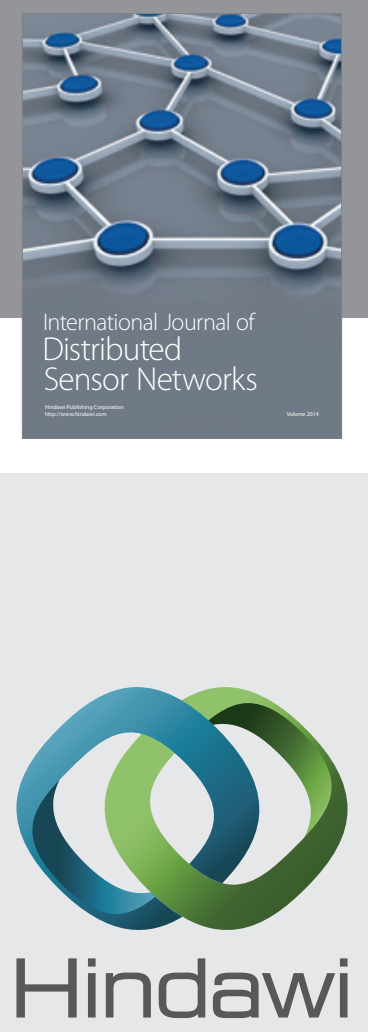

Submit your manuscripts at

http://www.hindawi.com
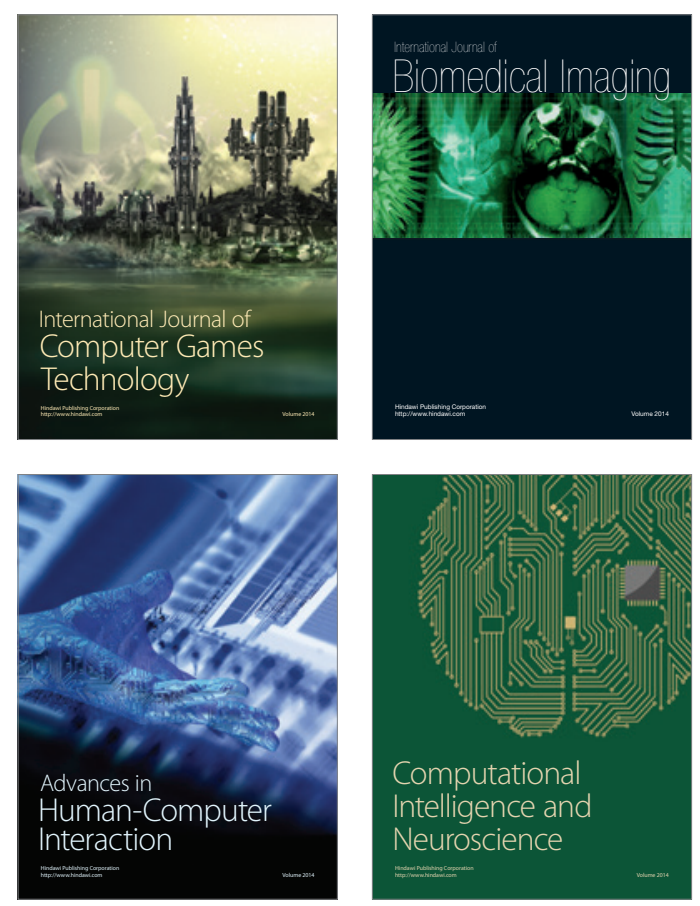
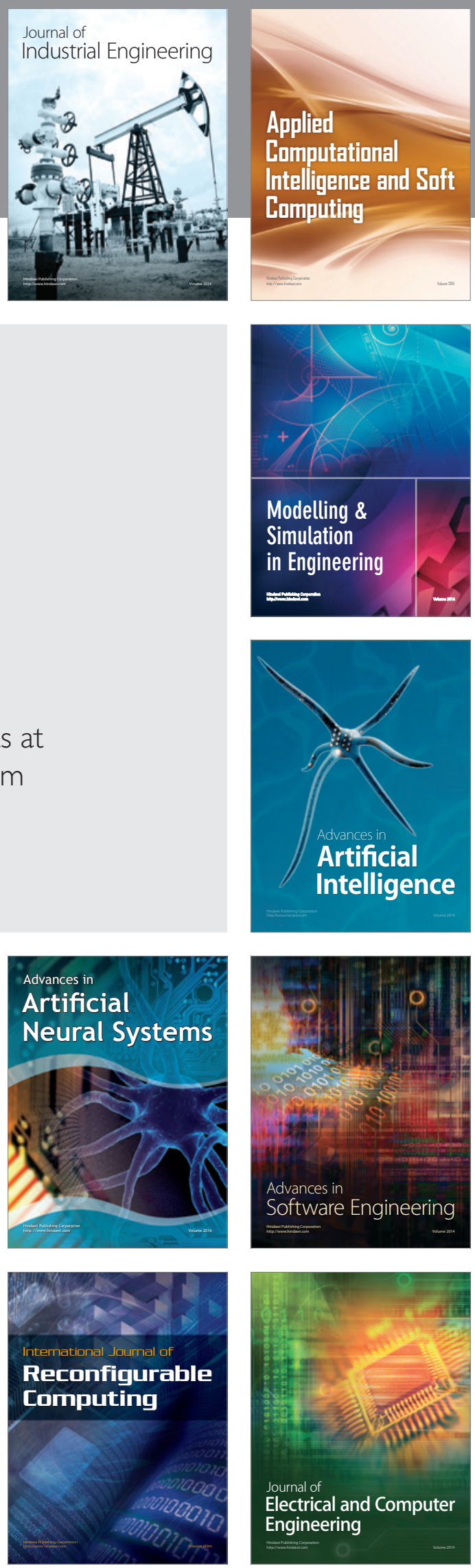